\title{
Constructive Near-Field Interference Effect in a Birdcage MRI Coil with an Artificial Magnetic Shield
}

\author{
K. Lezhennikova, ${ }^{1}$ R. Abdeddaim, ${ }^{2,}{ }^{*}$ A. Hurshkainen, ${ }^{1}$ A. Vignaud, ${ }^{3}$ M. Dubois,,${ }^{2,4}$ P. Jomin, ${ }^{2}$ \\ D. Berrahou, ${ }^{5}$ A. Raaijmakers, ${ }^{6}$ N. Avdievich, ${ }^{7}$ I. Melchakova, ${ }^{1}$ S. Enoch $\odot,{ }^{2}$ P. Belov, ${ }^{1}$ \\ C. Simovski, ${ }^{1,8}$ and S. Glybovski ${ }^{1}$ \\ ${ }^{1}$ Department of Physics and Engineering, ITMO University, St. Petersburg, Russia \\ ${ }^{2}$ Aix Marseille Université, CNRS, Centrale Marseille, Institut Fresnel, Institut Marseille Imaging, \\ Marseille, France \\ ${ }^{3}$ Université Paris-Saclay, CEA, CNRS, BAOBAB, NeuroSpin, 91191 Gif-sur-Yvette, France \\ ${ }^{4}$ CNRS, CRMBM, Aix Marseille Université, Marseille, France \\ ${ }^{5}$ Multiwave Imaging, Marseille, France \\ ${ }^{6}$ University Medical Center Utrecht, Utrecht, Netherlands \\ ${ }^{7}$ Max Planck Institute for Biological Cybernetics, Tübingen, Germany \\ ${ }^{8}$ Department of Electronics and Nanoengineering, Aalto University, Aalto, Finland
}

(Received 16 January 2020; revised manuscript received 12 April 2020; accepted 28 April 2020; published 2 June 2020)

\begin{abstract}
Radio-frequency (rf) coils are used in all clinical and research magnetic-resonance-imaging (MRI) systems to excite nuclear spins and to receive signals from them. The quality of imaging depends strongly on the signal-to-noise ratio (SNR) and the transmit efficiency of the coils. The birdcage volume coils used in most MRI scanners for homogeneous imaging of a sample are typically shielded from the external systems of the MRI scanner, i.e., the gradient coils, to confine the rf field within the region of interest. However, the near magnetic field of a conventional copper rf shield surrounding a birdcage coil interferes destructively with the primary field of the coil in the sample, which significantly limits the SNR and the transmit efficiency. In the work presented here, we theoretically study and experimentally demonstrate the possibility of creating an artificial magnetic rf shield for a birdcage coil with constructive interference in a sample. This effect is similar to the in-phase reflection of antenna far fields from a magnetic shield but affects the near field in MRI. We build an analytical model of a birdcage coil shielded with a cylindrical impedance boundary and analyze the conditions for increasing the efficiency of the coil by means of the shield. We conclude that by replacing a copper shield with an artificial magnetic one, it is possible to reduce the dissipative intrinsic losses of the coil and increase the power absorbed by the sample, which improves the efficiency. To demonstrate the effect, we perform a detailed numerical simulation and an experiment with a small birdcage in a 7-T ${ }^{19} \mathrm{~F}$ MRI system with a magnetic shield implemented as a periodic cylindrical metal structure with corrugations filled with a ceramic.
\end{abstract}

DOI: 10.1103/PhysRevApplied.13.064004

\section{INTRODUCTION}

The quality of magnetic resonance imaging (MRI) depends strongly on radio-frequency (rf) fields created by coils, that is, antennas specially designed to operate in the presence of a strongly conductive sample (the body to be imaged). In particular, the resolution of MRI requires a high signal-to-noise ratio (SNR). This means that the signal from the sample must be distinguished from the thermal noise produced by both the sample and the coil. The intrinsic coil noise is associated with dissipation losses in the metal and dielectric components of the coil and

\footnotetext{
*redha.abdeddaim@fresnel.fr
}

needs to be minimized. The best SNR is achieved when the noise comes mostly from the sample [1]. However, it is complicated to achieve this condition in many MRI applications. In particular, for so-called volume coils that image the entire sample, the latter may occupy only a small part of the coil. In this case, the intrinsic noise of the coil can dominate, leading to a poor SNR. For a single transceive coil, due to the reciprocity principle, the SNR is proportional to the transmit efficiency. For a linearly polarized field, the efficiency is defined as

$$
\eta=|H| / \sqrt{P_{\mathrm{acc}}},
$$

where $|H|$ is the magnitude of the rf magnetic field created by the coil in the transmit mode in the center of 
the sample, and $P_{\text {acc }}$ is the power accepted from the transmitter. Commonly, clinical and research MRI systems employ volume coils based on so-called birdcage resonators for $\mathrm{rf}$ excitation [2]. Volume coils can also be used in a transceiver mode, that is, for both transmission and reception. The birdcage resonator shown in Fig. 1(a) is based on a periodic cylindrical arrangement of parallel straight conductors (rungs) with two end rings, in which capacitors are connected periodically. This structure supports a slow wave propagating around its circumference $[3,4]$. In the low-frequency approximation (when the diameter of the resonator is electrically small), its two polarization-degenerate fundamental modes produce homogeneous linearly polarized magnetic fields in the whole cylinder volume. When driven from two ports in quadrature, the coil produces a homogeneous circularly polarized magnetic field. Birdcage coils can be efficient only when properly loaded with a conductive sample. It has been shown [1] that the efficiency [Eq. (1)] is related to the ratio between the unloaded $\left(Q_{U}\right)$ and loaded $\left(Q_{L}\right)$ quality factors of the resonator. $Q_{U}$ is the quality factor of the empty coil, while $Q_{L}$ is that with a sample in the coil. Poor loading, with small and weakly conducting samples, is associated with $Q_{U} / Q_{L} \approx 1$. In this case, the intrinsic loss dominates over the sample loss, and the coil becomes inefficient. The desired situation is $Q_{U} / Q_{L} \gg 1$, in which the coil is properly loaded and its efficiency is maximized.

Another issue that reduces the efficiency of birdcage coils is shielding. Typically, birdcages are surrounded by a cylindrical copper shield with an electrically small gap to isolate the coil from the gradient system, reduce radiation losses, and make their performance stable [5].

From skin-depth theory, a copper shield can be characterized by a complex surface impedance with a very low absolute value $Z_{C}=(1+i) \sqrt{\mu_{0} \omega / 2 \sigma_{s}}$, where $\sigma_{s}$ is the conductivity of copper, $\omega=2 \pi f$ is the angular frequency, and $\mu_{0}$ is the permeability of free space [6]. This boundary condition requires an almost zero tangential $E$ field component at the shield, and so the shield reflects electromagnetic waves out of phase and causes significant alteration of the primary magnetic and electric fields of the birdcage coil. The total field can be considered as the sum of a primary field due to currents in the coil and a field due to their image currents, representing the shielding effect. As a result, both the electric and the magnetic fields inside the coil are sharply reduced, increasing within the gap between the coil and the shield [7]. In the quasistatic approximation, this field reduction in the center of the coil reads $1-R_{\text {coil }}^{2} / R_{\text {shield }}^{2}$, where $R_{\text {coil }}$ and $R_{\text {shield }}$ are the radii of the coil and the shield, respectively [8]. This field redistribution effect may considerably decrease the efficiency.

An evident way to mitigate the destructive interference between the birdcage and the shield is to modify the surface impedance of the shield. From antenna theory, it is well known that for radiative fields, destructive interference can be even turned into constructive interference when the surface impedance is switched from zero to infinity (for a perfect magnetic conductor, PMC) [9]. In the case of a birdcage resonator, the current in the conductors of the coil induces an in-phase image current in a surrounding PMC shield. Hence, there are no field concentrations in the gap, and the field level inside the coil (i.e., in the sample) is expected to be almost independent of the gap width $R_{\text {shield }}-R_{\text {coil }}$.

Image magnetic currents do not exist in nature, but the behavior of PMC shields can be approximated using resonant periodic structures. Anisotropic PMC operation for both polarizations of an incident plane wave can be achieved using a mushroom-type high-impedance structure [10]. The effect of high-impedance shields, on which the tangential $H$-field component becomes small, on the radiation patterns of simple wire antennas has been investigated [11,12]. High-impedance surfaces have been used to design miniaturized reflectarrays [13], leaky-wave antennas [14], and absorbers [15,16], but their most popular application has been in artificial magnetic shields (AMSs) for low-profile unidirectional antennas. As has been shown in the literature, AMSs allow placing an antenna with a horizontal polarization, e.g., a dipole [17] or a microstrip patch [18], at an electrically small distance from the plane
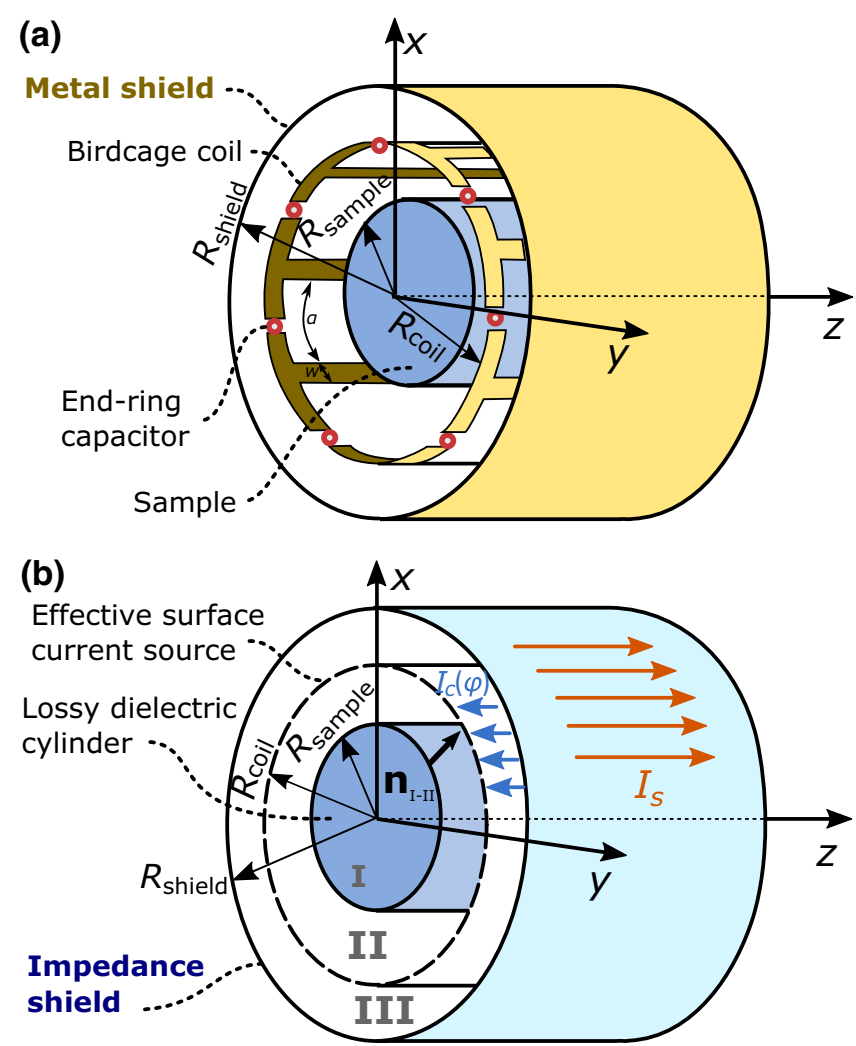

FIG. 1. Real geometry (a) and homogenized two-dimensional model (b) of a birdcage coil with a conductive cylindrical sample. 
of the shield. Unlike the case for a metal shield, at the resonance of the AMS, the antenna can approach the shield with a very narrow gap without its radiation efficiency being damaged, due to constructive interference [17]. Furthermore, AMSs and similar resonant structures have been used as walls for microwave cavities and waveguides to allow miniaturization of them and below-cutoff propagation $[19,20]$.

Recently, the approach of using AMSs has been applied to individually driven dipole elements in a head array for use at $7 \mathrm{~T}$ [21]. The dipoles provided a 7\% higher rf magnetic field in the center of a sample compared with a copper shield for the same overall accepted power, although the mutual coupling between the dipoles was increased. Moreover, a high-impedance surface has been applied as a shield for a surface-loop coil in a 7-T MRI system, also improving its field level [22].

One practical realization of a PMC is in the form of a corrugated surface [23]. This structure, with grooves of a quarter-wave depth, behaves like a perfect magnetic conductor for one linear polarization of the incident waves. Corrugated surfaces can be miniaturized and made into electrically thin structures by filling the grooves with a high-permittivity dielectric material [24].

In this paper, we study analytically the near-field effect of a PMC on the performance of a birdcage coil. Based on the analytical model, we investigate the possibilities for improving the efficiency. The possibility of using AMSs with a birdcage coil has not been previously studied. A birdcage itself is a complex multimode resonator. As we show in this paper, the presence of a resonant AMS considerably changes the resonant frequencies and fields of the different eigenmodes of the birdcage, including its fundamental mode. However, in an appropriate regime of coupled oscillations, the AMS provides constructive interference with the birdcage and an improvement in its field level [25]. In Sec. II, we analytically model a birdcage in the presence of a PMC to investigate the possibilities for improving the efficiency of the coil. After that, in Sec. III A, we investigate numerically the corresponding regime of near-field constructive interference between the coil and the AMS based on a periodically corrugated surface filled with a ceramic. Finally, we demonstrate that our AMS behaves similarly to a PMC and allows one to improve the efficiency and loading of a birdcage, as confirmed experimentally in Sec. III B.

\section{THEORY OF BIRDCAGE COIL WITH AN ARBITRARY IMPEDANCE SHIELD}

\section{A. Approximate analytical model}

To simplify the analysis of a birdcage in the presence of an AMS, we start with an approximate analytical description, which is, however, capable of predicting the possible benefits in terms of efficiency, and the conditions required to achieve them. In all expressions, we use harmonic time variations of the form $\exp (j \omega t)$.

It is well known that linear currents flowing in the straight conductors (rungs) of a birdcage at the resonance of its fundamental mode have a sinusoidal distribution around the circumference, with the total phase shift being equal to $360^{\circ}$. In other words, the current in the $n$th conductor, at an axial angle $\phi_{n}$, as shown in Fig. 1(a), reads $I_{n}=$ $I_{0} \cos \left(\phi_{n}\right) g(z)$, where $I_{0}$ is the common complex magnitude of the mode, and $g(z)$ is a smooth function describing the current distribution along the $z$ axis, which depends mainly on the coil length $L ; n=0,1, \ldots, N-1$, with $N$ being the number of rungs. If the period of the rungs $2 \pi R_{\text {coil }} / N$ is much smaller than the coil radius $R_{\text {coil }}$, the field of the fundamental mode can be calculated using a continuous surface current distribution instead of discrete currents [26]:

$$
I_{c}=I_{0} \cos (\varphi) g(z) .
$$

To qualitatively study the effect of an impedance shield on the field of a birdcage, we consider the two-dimensional model shown in Fig. 1(b), in which the coil is infinite in the $z$ direction $[g(z)$ is constant], while the source is given by Eq. (2).

The model consists of the cylindrical surface current described above, an impedance shield, and a conductive circular cylinder representing the sample, with relative permittivity $\varepsilon_{r}$ and conductivity $\sigma$. In our model, we neglect the end-ring currents and allow all currents to flow only in the $z$ direction, which means that we consider only a TM-polarized field. In particular, there is only a nonzero longitudinal electric field component $E_{z}$, while the magnetic field has only two transverse components, $H_{\phi}$ and $H_{\rho}$. To calculate the field components due to the given current source, we set boundary conditions on coaxial cylindrical surfaces as follows. The shield is defined by an impedance boundary condition, where the tangential component of the electric field is proportional to the induced surface current, i.e., $I_{s} Z_{s}=E_{z}$ at $\rho=R_{\text {shield, }}$, with an arbitrary surface impedance $Z_{s}$. Both $E_{z}$ and $H_{\phi}$ must be continuous at the air-dielectric boundary at $\rho=R_{\text {sample. }}$. In each of three volumes I, the sample $\left(\rho<R_{\text {sample }}\right)$, II, the space between the sample and the coil $\left(R_{\text {sample }}<\rho<R_{\text {coil }}\right)$, and III, the gap between the coil and the shield ( $\left.R_{\text {coil }}<\rho<R_{\text {shield }}\right)$, $E_{z}$ satisfies the Helmholtz equation,

$$
\frac{1}{\rho} \frac{\partial}{\partial \rho} \rho \frac{\partial E_{z}}{\partial \rho}+\frac{1}{\rho^{2}} \frac{\partial^{2} E_{z}}{\partial \varphi^{2}}+k^{2} E_{z}=0,
$$

where $k=k_{0}=\omega / c$ is the wave number in free space and $k=k_{1}=k_{0} \sqrt{\hat{\varepsilon}_{r}}$ is that in the sample medium, with complex permittivity $\hat{\varepsilon}_{r}=\varepsilon_{r}-j \sigma / \varepsilon_{0} \omega$. Equation (3) is solved 
by separation of variables:

$$
E_{z}^{i}=\sum_{m=0}^{\infty}\left[A_{m}^{i} J_{m}(k \rho)+B_{m}^{i} Y_{m}(k \rho)\right]\left[\begin{array}{c}
\cos m \varphi \\
\sin m \varphi
\end{array}\right],
$$

where $J_{m}(k \rho)$ and $Y_{m}(k \rho)$ are Bessel functions of the first and the second kind, respectively, of order $m$, and $A_{m}^{i}, B_{m}^{i}$ are complex coefficients to be determined for the three volumes $i=\mathrm{I}$, II, III.

The given current source flowing in the cylindrical surface of the coil ( $\rho=R_{\text {coil }}$ ) can be taken into account by writing the boundary condition as a jump in the tangential component of the magnetic field equal to $H_{\varphi}^{\mathrm{III}}-H_{\varphi}^{\mathrm{II}}=I_{c}$. The corresponding tangential component of the electric field remains continuous:

$$
E_{\varphi}^{\mathrm{III}}=E_{\varphi}^{\mathrm{II}} .
$$

Because of the symmetry of the problem and the particular shape of the excitation, $\cos \phi$, the field in each volume has the same angular dependence (only one fundamental-mode contribution is kept),

$$
E_{z}^{i}=\left[A^{i} J_{1}(k \rho)+B^{i} Y_{1}(k \rho)\right] \cos \varphi .
$$

The magnetic field components can be derived from Eq. (5) using the Maxwell equation $\nabla \times \mathbf{E}=-j \omega \mu_{0} \mathbf{H}$. After substituting all the field components into the five boundary conditions, and given that $E_{z}$ must have no singularity at $\rho=0$ ( $B^{i}$ is zero), only five unknowns are left to be found. This is done by solving the matrix equation

$$
[A]=[M]^{-1} \cdot[I],
$$

where $[A]$ is the vector of the unknown coefficients, $[I]$ is a vector depending on the source currents of the birdcage, and $[M]$ is a $5 \times 5$ matrix with coefficients expressed through Bessel functions depending on the coil parameters, including the shield impedance. The expressions for $[I]$ and $[M]$ are given in the Appendix.

Based on the fields calculated using the unknowns once they have been determined, it is possible to characterize the efficiency of the coil using Eq. (1). Assuming that all power accepted from the transmitter is dissipated in the sample, i.e., the coil is lossless $\left(P_{\text {acc }}=P_{\text {sample }}\right)$, the efficiency can be calculated as the volume integral of the power-loss density $[1,27]$ :

$$
\eta=\frac{|H(\rho=0)|}{\sqrt{P_{\text {sample }}}}=\frac{\lim _{\rho \rightarrow 0}\left|H_{\rho}\right|}{\sqrt{\frac{1}{2} \int_{0}^{2 \pi} \int_{0}^{R_{\text {sample }}} \sigma\left|E_{z}\right|^{2} \rho d \rho d \varphi}} .
$$

Since $B^{I}$ is zero, both the electric and the magnetic field in the sample volume are proportional to the same coefficient $A^{I}$, which in turn depends on the shield impedance. As a result, the efficiency in Eq. (8) does not depend on $Z_{S}$. After some simple derivations, it can be shown that the efficiency depends only on the size and material parameters of the sample and is determined by the expression

$$
\eta=\frac{\left(c \mu_{0} R_{\text {sample }}\right)^{-1}\left|\sqrt{\hat{\varepsilon}_{r}}\right|}{\sqrt{\pi \sigma\left[J_{1}^{2}\left(k_{1} R_{\text {sample }}\right)-J_{0}\left(k_{1} R_{\text {sample }}\right) J_{2}\left(k_{1} R_{\text {sample }}\right)\right]}} .
$$

This allows us to conclude that if all transmit power is absorbed in the sample, the shield impedance does not affect the shape of the distribution of either the $E$ or the $H$ field within the sample, and, consequently, it does not affect the efficiency. In fact, the shield impedance dramatically affects the ratio between the magnetic field and the current: if a metal shield is replaced by a PMC one, this ratio is increased. However, the electric field also increases proportionally to the given source current, which holds the efficiency at the same level. Therefore, if a volume coil is properly loaded (all input power is absorbed by the sample and $Q_{L} \ll Q_{U}$ ), it cannot be improved by using a magnetic shield. However, as shown in the following, if the coil is insufficiently loaded by the sample and intrinsic coil losses are relevant, an AMS can improve the efficiency.

It is possible to extend our model by taking into account the losses in the birdcage rungs as well as the losses in the impedance shield. The boundary condition on the shield already contains an arbitrary surface impedance, which, for the reference coil, is equal to the surface impedance of the corresponding metal (e.g., copper). A PMC shield has an infinite surface impedance, while an AMS with losses has a high but finite purely real impedance at the resonance. So far, the given surface current $I_{c}$ has been equal to a step in the tangential magnetic field component, and this given source only generates power. In the case of a lossy coil, the total complex power due to the current $I_{C}$ contains generated, dissipated, and reactive power. The latter two types of power can be introduced by using a nonzero internal complex impedance in the distributed source. This impedance has the meaning of a surface-averaged complex grid impedance $Z_{\text {coil }}$ of the periodic structure of copper birdcage rungs. Thus the total complex power per unit length of the coil in the $z$ direction at a given current density [Eq. (2)] reads

$$
\dot{P}=-\frac{1}{2} \int_{0}^{2 \pi} E_{z} I_{C}^{*} R_{\text {coil }} d \varphi+\frac{1}{2} \int_{0}^{2 \pi}\left|I_{C}\right|^{2} Z_{\text {coil }} R_{\text {coil }} d \varphi .
$$

The impedance response of a real coil, which is in practice a discrete structure of $N$ thin copper strips of width $w$ periodically arranged on a cylindrical surface with a period of $\alpha=2 \pi R_{\text {coil }} / N$, can be determined according to the Kontorovich method of averaged boundary conditions $[28,29]$ 
as

$$
Z_{\text {coil }}=Z_{w} \alpha+j \eta_{0} \frac{\alpha}{\lambda_{0}} \log \frac{\alpha}{2 \pi r_{w}}
$$

where $r_{w}$ is the effective radius of a thin strip conductor of width $w$ [30] $\left(r_{w}=w / 4\right)$, and $Z_{w}$ is the linear impedance of a circular wire. In the approximation in which the thickness of the conductor is large compared with the skin depth, the impedance of the wire can be calculated as $Z_{w}=(1+j) / \delta \pi r_{w} \sigma_{s}$, where $\delta=\sqrt{2 / \omega \sigma_{s} \mu_{0}}$ is the skin depth and $\sigma_{s}$ is the conductivity of the birdcage rungs. The expression for the complex power [Eq. (10)] can be conveniently rewritten as

$$
\begin{aligned}
\dot{P} & =\frac{\pi|I|^{2}}{2}\left(-\frac{E_{z}^{\mathrm{II}}\left(\rho=R_{\text {coil }}\right)}{I}+Z_{\text {coil }}\right) \\
& =\dot{P}_{\text {sample }}+\dot{P}_{\text {coil }}+\dot{P}_{\text {shield }},
\end{aligned}
$$

where $E_{z}^{\mathrm{II}}\left(\rho=R_{\text {coil }}\right)=A^{\mathrm{II}} J_{1}\left(k_{0} R_{\text {coil }}\right)+B^{\mathrm{II}} Y_{1}\left(k_{0} R_{\text {coil }}\right)$ is the tangential component of the electric field on the surface of the coil, $P_{\text {coil }}$ is the complex power due to the coil rungs, $P_{\text {sample }}$ is the power absorbed by the sample, and $P_{\text {shield }}$ is the complex power due to the shield. All parts of the power are assumed to be per unit length in the $z$ direction. The complex power $\dot{P}_{\text {shield }}$ can be calculated based on the previously found field components as

$$
\dot{P}_{\text {shield }}=\frac{\pi R_{\text {shield }}}{2 Z_{\text {shield }}^{*}}\left|A^{\mathrm{III}} J_{1}\left(k_{0} R_{\text {shield }}\right)+B^{\mathrm{III}} Y_{1}\left(k_{0} R_{\text {shield }}\right)\right|^{2} .
$$

Equations (12) and (13) allow us to determine separately, for a given surface current, the power absorbed in the sample as

$$
P_{\text {sample }}=\mathfrak{R}(\dot{P})-\mathfrak{R}\left(\dot{P}_{\text {shield }}\right)-\mathfrak{R}\left(\dot{P}_{\text {coil }}\right)
$$

and the total intrinsic dissipated power in the coil as

$$
P_{\text {loss }}=\mathfrak{R}\left(\dot{P}_{\text {coil }}\right)+\mathfrak{R}\left(\dot{P}_{\text {shield }}\right) .
$$

We assume the birdcage rungs and the conventional shield in the reference case to be made of copper with a conductivity of $\sigma_{s}=59.5 \mathrm{~S} / \mathrm{m}$. As discussed above, the efficiency of a birdcage in the absence of intrinsic losses does not depend on the shield surface impedance and should be the same in the cases of rf electric and magnetic shields: $\eta^{\text {elec }}=\eta^{\text {magn }}$. Therefore, one can write

$$
\eta^{\text {magn }}=\frac{\left|H^{\text {magn }}(\rho=0)\right|}{\sqrt{P_{\text {sample }}^{\text {magn }}}}=\frac{\left|H^{\text {elec }}(\rho=0)\right|}{\sqrt{P_{\text {sample }}^{\text {elec }}}}=\eta^{\text {elec }} .
$$

With the same source current, in the presence of losses, the same signal levels $\left|H^{\text {elec }}(\rho=0)\right|$ and $\left|H^{\text {magn }}(\rho=0)\right|$ are provided as for the lossless coil, but the total power consumed is changed, and so the efficiency levels become different:

$$
\begin{aligned}
\eta^{\text {magn }} & =\frac{\left|H^{\text {magn }}(\rho=0)\right|}{\sqrt{P_{\text {sample }}^{\text {magn }}+P_{\text {loss }}^{\text {magn }}}} \\
& \neq \frac{\left|H^{\text {elec }}(\rho=0)\right|}{\sqrt{P_{\text {sample }}^{\text {elec }}+P_{\text {loss }}^{\text {elec }}}}=\eta^{\text {elec }} .
\end{aligned}
$$

On the basis of the last two expressions, it is easy to show that the efficiency gain $G=\eta^{\text {magn }} / \eta^{\text {elec }}$ due to replacement of an electric shield with a magnetic one is

$$
G=\sqrt{\frac{1+P_{\text {loss }}^{\text {elec }} / P_{\text {sample }}^{\text {elec }}}{1+P_{\text {loss }}^{\text {magn }} / P_{\text {sample }}^{\text {magn }}}} .
$$

The best possible gain is achieved for an initially weakly loaded coil with high intrinsic dissipation losses. By replacing the metal shield with a PMC one, one can make the power dissipated in the sample much larger than the intrinsic loss power of the coil. In this scenario, the best efficiency gain can be estimated from our theoretical model as

$$
G_{\max }=\sqrt{1+P_{\text {loss }}^{\text {elec }} / P_{\text {sample }}^{\text {elec }}}
$$

As can be seen from the last expression, the efficiency gain is limited by the initial ratio between the levels of the intrinsic loss and the sample power. Therefore, an AMS is worth applying in the case of birdcage coils with weak loading, e.g., due to a small sample. In other words, the closer the loaded and unloaded quality factors of the birdcage are to each other, the higher the efficiency improvement.

To compare the efficiency of a birdcage coil with a copper shield and the same coil with a PMC shield, let us consider a small birdcage configuration for preclinical MRI at 7 T. In this application, small animals (e.g., a rat or a mouse) of very different sizes can be used as samples. Using our model, it is possible to study how the efficiency gain depends on the diameter of a lossy cylindrical sample and on the frequency. In efficiency-gain calculations based on the formula in Eq. (18), for the sample, we use the following dielectric parameters of a commercially available liquid for calibration of body coils: $\varepsilon_{r}=$ $58, \sigma=0.95 \mathrm{~S} / \mathrm{m}$. The same liquid is also used in measurements. The coil radius $R_{\text {coil }}$ is assumed to be $36 \mathrm{~mm}$, which corresponds to a commercially available transmitreceive birdcage coil for a 7-T preclinical scanner made by Bruker [31].

In Fig. 2(a), the calculated gain is shown versus the sample diameter and the frequency for a shield radius 


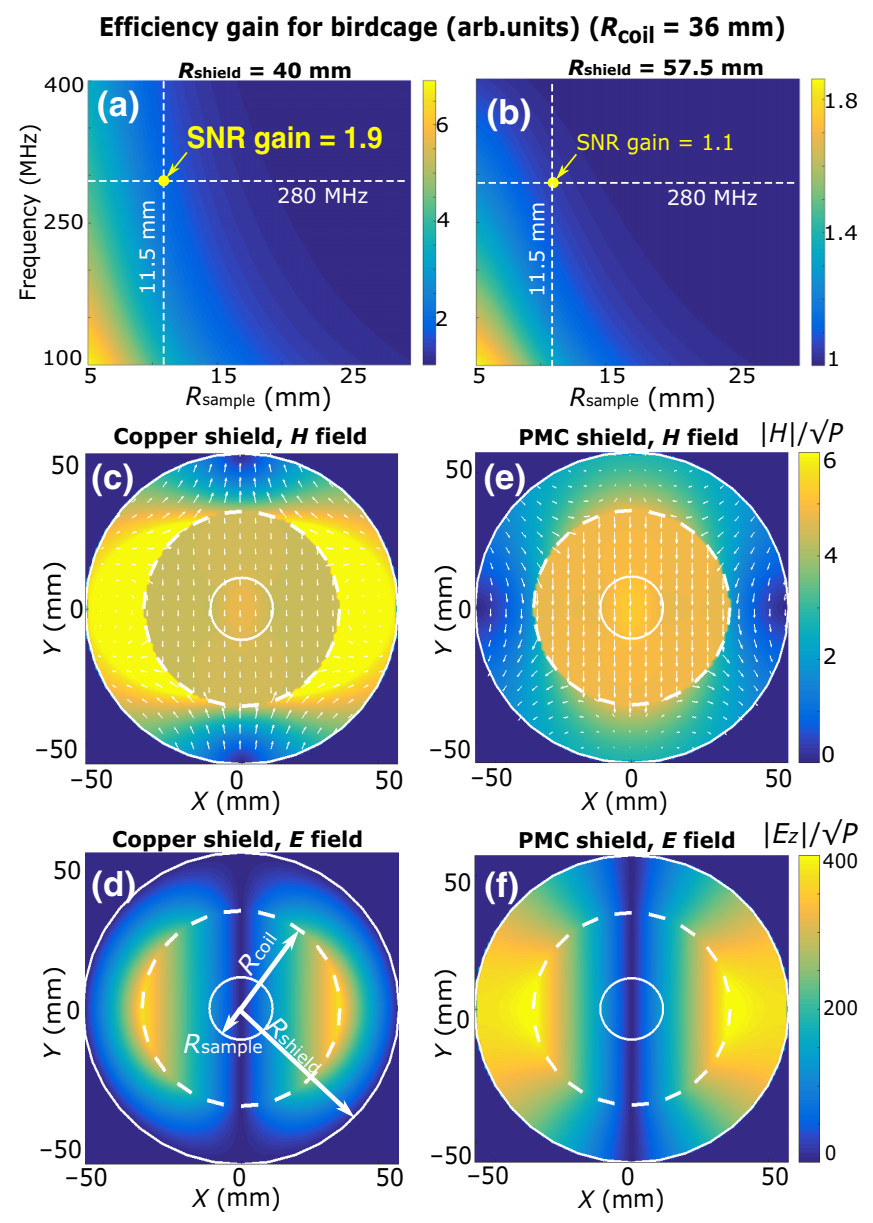

FIG. 2. Analytically calculated efficiency gain due to an ideal magnetic shield (PMC) for a preclinical birdcage coil operating at $7 \mathrm{~T}(280 \mathrm{MHz})$ : (a) $R_{\text {shield }}=40 \mathrm{~mm}$; (b) $R_{\text {shield }}=57.5 \mathrm{~mm}$. Analytically calculated maps of magnetic field (c),(e) and electric field (d),(f) for $R_{\text {shield }}=57.5 \mathrm{~mm}$ with a copper (left) and a PMC (right) shield.

$R_{\text {shield }}=40 \mathrm{~mm}$. To show that the radius of a copper shield can strongly affect the performance of the coil, a similar dependence is presented for $R_{\text {shield }}=57.5 \mathrm{~mm}$ in Fig. 2(b) for comparison. The arrow indicates $280 \mathrm{MHz}$, which is the Larmor frequency of ${ }^{19} \mathrm{~F}$ nuclei at $7 \mathrm{~T}$, and the sample radius is $11.5 \mathrm{~mm}$. A sample of this size corresponds roughly to the dimensions of a laboratory mouse. As can be seen from a comparison of the plots in Figs. 2(a) and 2(b), the smaller the shield radius and the narrower the gap between the shield and the coil, the higher the efficiency gain. A narrower metal shield makes the birdcage less efficient due to destructive interference. However, with the PMC shield, this is not the case. Moreover, one can observe in Figs. 2(a) and 2(b) that the magnetic shield improves the efficiency better at lower frequencies, which can be explained by a reduction of the effect of losses in the sample compared with losses in the coil conductors. Finally, for the same reason, the smaller the sample, the higher the gain is.

The distributions of the electric and magnetic fields in a transverse section of the coil for a copper shield of radius $R_{\text {shield }}=57.5 \mathrm{~mm}$ are depicted in Figs. 2(c) and 2(d), and corresponding distributions for a PMC shield are depicted in Figs. 2(e) and 2(f). The fields are normalized by the square root of the accepted power per unit length in the $z$ direction. This power is calculated as a volume integral of the power-loss density within the sample based on the $E$-field distribution. In other words, the absolute values of the $H$ field in Figs. 2(c) and 2(e) are proportional to the efficiency according to Eqs. (16) and (17).

From a comparison of the analytically calculated field maps, it is clear that replacing a copper shield with a PMC one changes the magnetic and electric field distributions. In particular, in the presence of a PMC shield, the magnetic field for the same accepted power becomes weaker in the gap between the coil and the shield, while it increases in the center of the coil. This means that the magnetic shield increases the efficiency of the coil in the transmit mode. However, the shapes of the $E$ - and $H$-field distributions in the sample do not change; instead, they remain homogeneous.

\section{B. Numerical solution for a birdcage coil with an ideal rf magnetic shield}

The above conclusions are valid for a model (infinite) coil with a smooth distribution of surface current. To validate them for a realistic birdcage resonator, numerical simulations are performed. We consider a high-pass birdcage coil made of $N=8$ copper rungs, surrounded by a cylindrical PMC shield. The results are compared with the case of a conventional copper shield. All simulations are done using the frequency-domain solver in CST Microwave Studio 2017 (using the finite-element method). As an example, a preclinical small coil is considered, with the same radius $R_{\text {coil }}=36 \mathrm{~mm}$ as in the analytical calculations. The length of the rungs is taken as $110 \mathrm{~mm}$. In all simulations, a single discrete port is placed in parallel with one of the end-ring capacitors for excitation of the birdcage, as shown in Figs. 1(a) and 3(a). With only one port, we compare the operation of the birdcage in the presence of different shields in the linear-polarization regime. In all cases considered in this section, the coil is tuned to $280 \mathrm{MHz}$ by adjusting the capacitors in the end-ring conductors. In the simulations, the sample is represented by a homogeneous dielectric cylinder with a length of $80 \mathrm{~mm}$ and the same material properties as in the analytical model.

First, we study the dependence of the efficiency on the shield radius for a sample radius of $11.5 \mathrm{~mm}$. The results are shown for a copper and a magnetic shield in Fig. 4(a). 

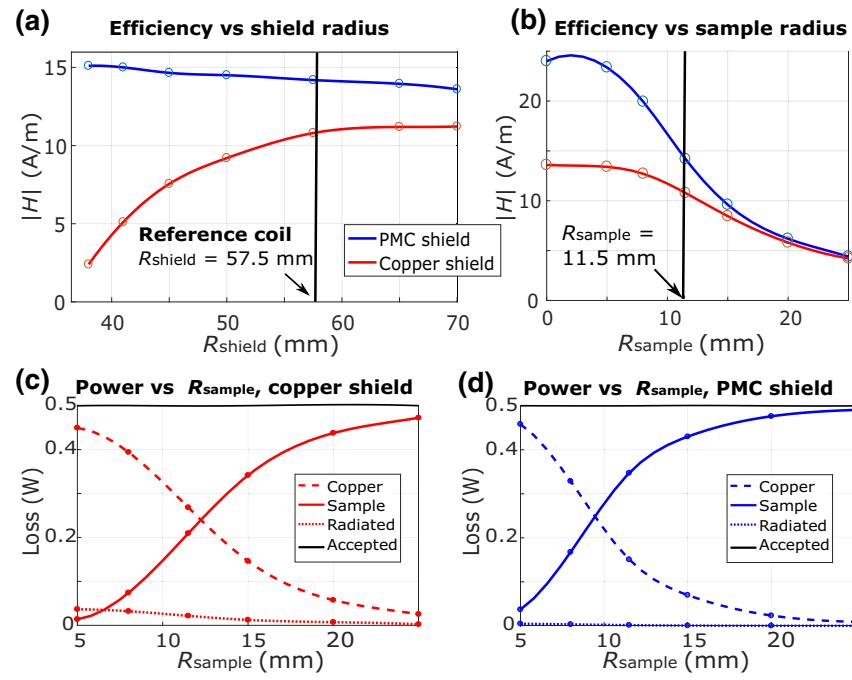

(e)

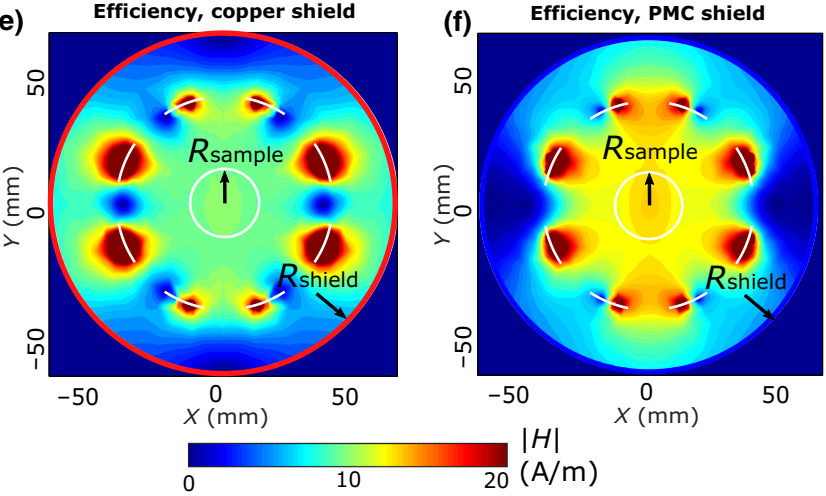

FIG. 3. Setup for simulation of a small birdcage inside an AMS (PISA): (a) cross section; (b) perspective view; (c) conventional planar corrugated structure filled with dielectric.

These results demonstrate that the efficiency of the birdcage with the PMC shield is almost independent of the size of the shield. This can be explained by constructive interference with in-phase mirror currents induced by the birdcage rungs in the shield. In contrast, the efficiency with a copper shield decreases rapidly when the shield approaches the coil (i.e., $R_{\text {shield }}$ becomes close to $R_{\text {coil }}=$ $36 \mathrm{~mm}$ ), due to destructive field interference. Noticeably, when the radius of the copper shield is changed, there is a point $R_{\text {shield }}=57.5 \mathrm{~mm}$ from which a further increase in the shield radius has almost no effect on the efficiency. So, for a copper shield, this is the smallest shield radius for which one can obtain the maximum efficiency. This value is taken for the reference coil to make comparisons with in the simulations and measurements.

Figure 4(b) shows the dependence of the efficiency of the same coil on the sample radius for a fixed $R_{\text {shield. }}$. It can be observed that the efficiency gain is higher for smaller sample sizes, and the gain is highest when the coil is empty (contains no sample). For the sample radius of $11.5 \mathrm{~mm}$ considered, the PMC shield gives a gain of $33 \%$. For larger

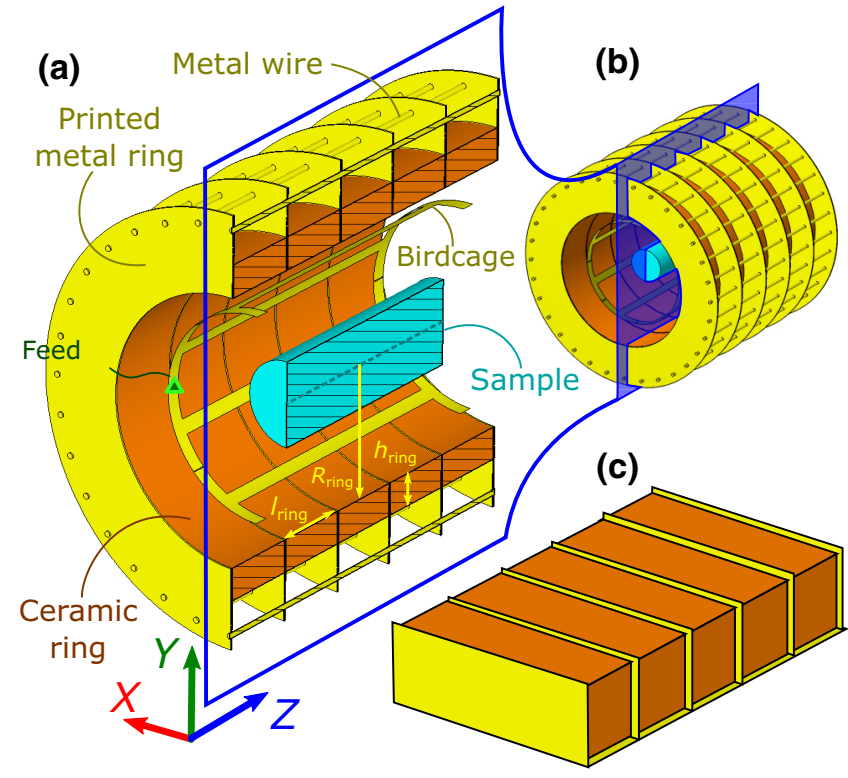

FIG. 4. (a),(b) Numerically calculated magnitudes of the magnetic field created by a birdcage coil at an accepted power of $0.5 \mathrm{~W}$ (i.e., efficiency): (a) level at the center of the sample for copper and PMC shields versus the shield radius; (b) level at the center of the sample for copper and ideal magnetic shields versus the sample radius. (c),(d) Power dissipated in the sample and the coil and radiated power with (c) copper shield and (d) PMC shield. $R_{\text {shield }}=57.5 \mathrm{~mm}$ and $R_{\text {sample }}=11.5 \mathrm{~mm}$. (e),(f) Distribution in transverse plane with (e) copper shield and (f) PMC shield.

radii of the sample, the gain due to the PMC shield is reduced, and becomes negligible when $R_{\text {sample }}>20 \mathrm{~mm}$. This means that, as in the analytical model, a PMC shield does not improve the efficiency of a properly loaded coil. Numerically calculated magnetic field patterns in the central transverse plane of the coil for an accepted power of $0.5 \mathrm{~W}$ are shown in Figs. 4(e) and 4(f) for a copper and a PMC shield, respectively. As in the analytically calculated plots, the magnetic shield concentrates most of the magnetic field energy within the coil, while in the case of the copper shield the concentration takes place in the gap between the coil and the shield. This difference in field patterns is similar to that obtained from the analytical model [compare Figs. 2(c) and 2(e) with Figs. 4(e) and 4(f)]. The efficiency gain observed in the simulations is due to a change in the ratio between the power absorbed by the sample and the power dissipated in the copper conductors of the coil. The plots given in Figs. 4(c) and 4(d) show that when the sample is small and the loading is weak $\left(Q_{U} / Q_{L} \approx 1\right)$, the power absorbed by the sample can be considerably increased by a PMC shield. In contrast, for large samples, almost all the accepted power of $0.5 \mathrm{~W}$ is absorbed by the sample no matter which shield is used. Therefore, as expected from Eq. (19), the magnetic shield improves the efficiency only when the coil is poorly loaded 
and initially a large portion of the power is dissipated within the coil. In terms of the receive mode, the role of the magnetic shield is to increase the signal received from the sample and also to increase the noise from the sample proportionally, but keeping the same intrinsic noise from the coil. As a result, the importance of the intrinsic noise decreases considerably, and the overall SNR increases. It should be noted that the quantitative gain predicted by Eq. (19) differs from that obtained from the numerical simulations ( $10 \%$ vs $30 \%)$ because our analytical model is approximate and only qualitatively describes the effect of the shield.

\section{IMPROVEMENT OF A BIRDCAGE MRI COIL WITH AN ARTIFICIAL MAGNETIC SHIELD: PROOF OF CONCEPT}

As follows from the results presented above, an ideal magnetic shield can improve the efficiency of an underloaded birdcage coil, for instance in the case of a small sample, when it replaces a conventional copper shield. To prove this effect experimentally, we design an AMS based on a cylindrical axially symmetric corrugated surface filled with a high-permittivity ceramic. The AMS, placed around a small birdcage coil, is shown in Figs. 3(a) and 3(b). A corrugated structure [Fig. 3(c)] is known to demonstrate high-impedance properties and in-phase reflection for incident waves polarized perpendicularly to the corrugations, at its quarter-wave resonance. Since the main component of the $E$ field of a birdcage is the $z$ component, the corrugations should be axial. In the AMS, we bend the corrugated surface so that it produces an axially symmetric structure with an internal radius $R_{\text {ring }}$ surrounding the birdcage to be improved.

\section{A. Design and numerical simulation of an artificial magnetic shield for a birdcage}

Hereinafter, we use the same birdcage as in the simulations presented the previous section, with the size of a commercial preclinical birdcage coil. We call this birdcage coil together with the above AMS, based on a cylindrical corrugated structure with axial symmetry, PISA (Preclinical Improved Signal Antenna). In the numerical simulations, the corrugations have thin copper walls and are partially filled with a dielectric with the material properties of a commercially available $\mathrm{CaTiO}_{3}$-based ceramic [32] $\left(\varepsilon_{r}=\right.$ $160, \tan \delta=0.0005)$. This is done by inserting ceramic rings into the corrugations as shown in Figs. 3(a) and 3(b). The inner radius of the dielectric ring is $R_{\text {ring }}=47 \mathrm{~mm}$, the thickness is $h_{\text {ring }}=11 \mathrm{~mm}$, and the length of every ring is $l_{\text {ring }}=26 \mathrm{~mm}$, which is equal to the period of the corrugations in the $z$ direction.

Although the conventional corrugated structure shown in Fig. 3(c) has a solid ground plane, a copper sheet connected to every wall of the corrugations, this design cannot be applied in MRI. This sheet is in fact incompatible because it supports unwanted eddy currents [33], excited during pulse sequences with rapidly changing gradient magnetic fields, which may cause imaging artifacts. To suppress eddy currents while keeping the same functionality at the Larmor frequency, we use a cylindrical grid of 32 parallel 2-mm-thick brass wires, as shown in Fig. 3(a). The wires are arranged with an axial periodicity around the ceramic rings at a radius of $70 \mathrm{~mm}$ and placed parallel to the birdcage rings by connecting each wire to all corrugation walls. The walls are $0.5-\mathrm{mm}$-thick copper rings. The number of wires is chosen to approximate the behavior of a solid metal shield, i.e., to confine the resonant field in each corrugation; this field can spread outside to a distance of approximately one interwire spacing. More wires would complicate the assembly of the AMS too much.

The birdcage itself is a multimode resonator, with a particular fundamental mode with maximum power dissipation in the sample at the corresponding resonance. For the high-pass birdcage considered here with a conventional copper shield, this mode has the highest frequency [3]. This resonant frequency can be tuned with the end-ring capacitors to the Larmor frequency. The dielectric-filled AMS also has a particular eigenmode, which has a magnetic field distribution and polarization similar to those of the fundamental birdcage mode. The two field distributions are shown in Fig. 5(a) together with numerically calculated values of $S_{11}$ at the birdcage port in the presence of
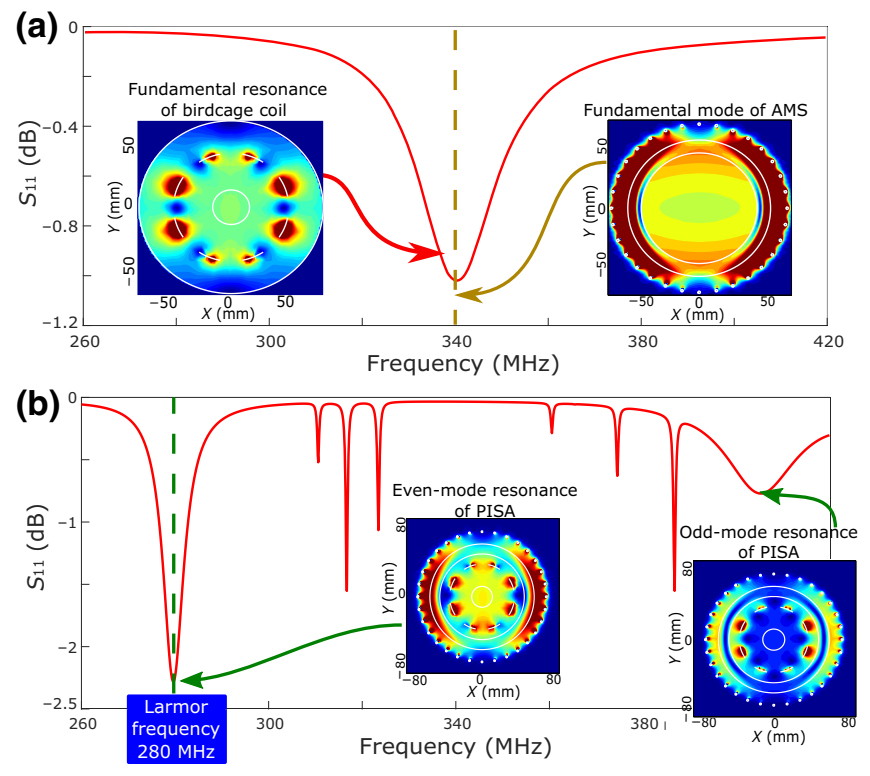

FIG. 5. Numerically calculated $S_{11}$ of birdcage coil, and magnetic field patterns in insets: (a) birdcage with copper shield (the resonant frequency and eigenmode field pattern of the AMS are additionally shown separately); (b) birdcage with AMS. 
a copper shield. In this case, the fundamental resonant frequencies of the birdcage and the AMS are both made close to $340 \mathrm{MHz}$. When the copper shield is replaced by the resonant AMS, the birdcage mode splits into two coupled modes: an even mode at $280 \mathrm{MHz}$ and an odd mode at $395 \mathrm{MHz}$. This hybridization effect occurs due to strong mutual coupling between the two resonators. The coupled mode resonances can be seen in Fig. 5(b) on the frequency curve of $S_{11}$ for the birdcage in the presence of the AMS. The corresponding field distributions are given in Fig. 5(b) as insets. Note that both the even and the odd modes have identical cosinelike field distributions over the angular coordinate and similar homogeneous profiles along the $Z$ axis. However, while the even mode has a maximum of the $H$ field in the center of the coil, the odd mode has a null at the same point. By comparison with Fig. 4(f), it is clearly seen that the effect of the even mode within the birdcage is similar to that of an ideal magnetic shield. In other words, the proposed structure provides constructive near-field interference at the even-mode resonance. In our case, this resonance is obtained at $280 \mathrm{MHz}$, which is the Larmor frequency of ${ }^{19} \mathrm{~F}$ fluorine nuclei in 7-T MRI.

In Fig. 6, we numerically compare the performance of a birdcage with a copper shield of radius $57.5 \mathrm{~mm}$ and PISA. Also, we compare the performance with results for an ideal PMC shield. All coils are tuned to $280 \mathrm{MHz}$ and matched to $50 \Omega$ with lumped capacitors at the input port using circuit cosimulation with CST Design Studio. Figure 6 shows the magnetic field patterns for a fixed accepted power of $0.5 \mathrm{~W}$ (the efficiency) for a sample of radius $11.5 \mathrm{~mm}$ and four different shields: an AMS without losses, an ideal PMC, an AMS with losses, and, finally, a copper shield. As can be seen from Fig. 6, for this sample, the field level obtained with the PMC shield is well above that with the copper shield (13.5 vs $10.8 \mathrm{~A} / \mathrm{m})$ for the same power, which means an efficiency improvement of $25 \%$. At the even-mode resonance of the lossless PISA, the efficiency level is the same as for the PMC shield. Comparing Figs. 6(a) and 6(b), one can conclude that PISA realizes the same efficiency improvement. Moreover, although the magnetic field is enhanced within the corrugated structure in the real resonant AMS, the shape of the field distribution inside the birdcage is the same as that obtained with the ideal PMC. Therefore, the effect of the AMS when its losses are neglected is the same as that of the PMC. When losses in the commercially available ceramic material of the rings and the finite conductivity of the metal elements of the corrugated structure are considered, the simulation still predicts an efficiency gain, though lower than for the PMC (it is only 13\%). Despite the reduced gain, the losses do not cancel the near-field constructive interference between the birdcage and the corrugated structure, and the gain is still observable.

In order to validate our numerical results, we check that the observed efficiency gain is consistent with the main
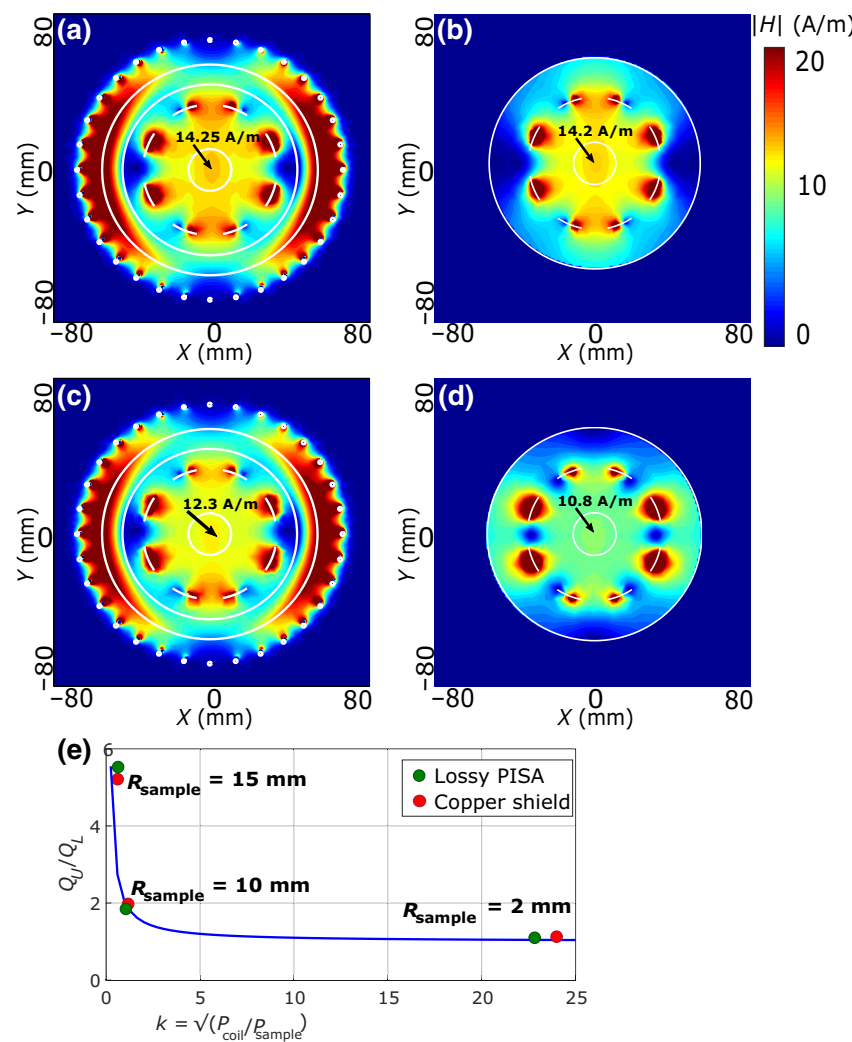

FIG. 6. Numerical comparison of the efficiency of a birdcage with different types of shield: magnitude of $H$ field for $0.5 \mathrm{~W}$ of accepted power in the central transverse plane. (a) Lossless AMS (PISA); (b) ideal PMC boundary; (c) AMS (PISA) with realistic material losses; (d) conventional copper shield; (e) ratio between unloaded and loaded quality factors of PISA with realistic material losses and for birdcage with conventional copper shield.

parameters of the loading, such as the ratio of the loaded to the unloaded quality factor and the ratio of the coil to the sample power. For any coil, the ratio $Q_{U} / Q_{L}$ has a simple relation with the ratio $P_{\text {coil }} / P_{\text {sample }}$ [34]. From the simulation, we determine the quality factors from the bandwidth of the resonance excited with a small test loop. The results are presented for PISA and for the birdcage with a copper shield in Fig. 6(e) as circles. In the same figure, the theoretical dependence is given by the blue curve. When $Q_{U} \approx Q_{L}$ (weak loading), the transmit power is redistributed in favor of the sample, and so the efficiency is improved. When the loading is strong $\left(Q_{U} / Q_{L} \geq 2\right)$, the efficiency is not improved.

\section{B. Experimental demonstration}

To experimentally demonstrate the efficiency improvement and the near-field interference effect investigated here, an AMS is fabricated and used as a shield for a small birdcage for 7-T MRI. For comparison, a conventional copper shield is built and combined with another instance 


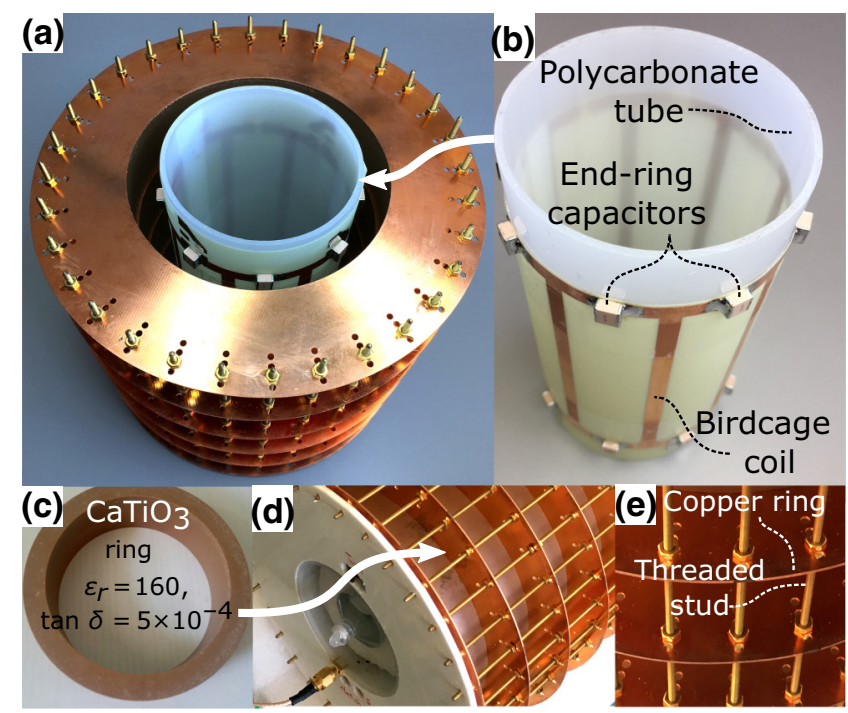

FIG. 7. Prototype of birdcage coil with AMS (PISA) (a) and its main parts: small high-pass birdcage with SMD end-ring capacitors (b); $\mathrm{CaTiO}_{3}$ ceramic ring (c); perspective view of assembled coil (d); thin-wire external ground-plane structure of corrugated surface (e).

of the same birdcage. The corrugated structure is formed from five ceramic rings of the same dimensions as in the simulations. The rings are specially manufactured from a $\mathrm{CaTiO}_{3}$ ceramic having the measured material properties $\tan \delta=0.0005$ and $\varepsilon_{r}=160$. The disks are separated by corrugation walls, realized as copper rings printed on both sides of Arlon $25 \mathrm{~N}$ 0.5-mm-thick substrates. The thin-wire structure on the periphery of the AMS contains 32 brass 2-mm-thick threaded studs, which are fixed to each copper ring of the corrugated structure using a pair of nuts. By tightening all nuts, each ceramic ring is fixed between two copper rings so that the whole AMS is mechanically robust. The birdcage, with the same geometry as in the simulations, is made of a bent 0.5 -mm-thick single-sided printed circuit board (PCB) on an FR4 substrate wrapped around a polycarbonate tube with an outer radius $R_{\text {coil }}=$ $36 \mathrm{~mm}$. The birdcage with the AMS and that with the conventional copper shield, of radius $57.5 \mathrm{~mm}$, are both tuned with soldered end-ring surface mount device (SMD) capacitors and driven through a coaxial cable connected in parallel with one of those capacitors. The assembled PISA and its parts are shown in Fig. 7.

To investigate the field distribution and efficiency of the empty birdcage in the presence of the AMS and the copper shield, the magnetic field is measured in the $Z X$ and $Z Y$ planes. The measurements are done by mechanically positioning a properly oriented small pickup-loop probe connected to a port of a vector network analyzer (VNA). The other port of the VNA drives the birdcage at one port (in the linear-polarization regime). The efficiency, proportional to the magnetic field normalized by the square root
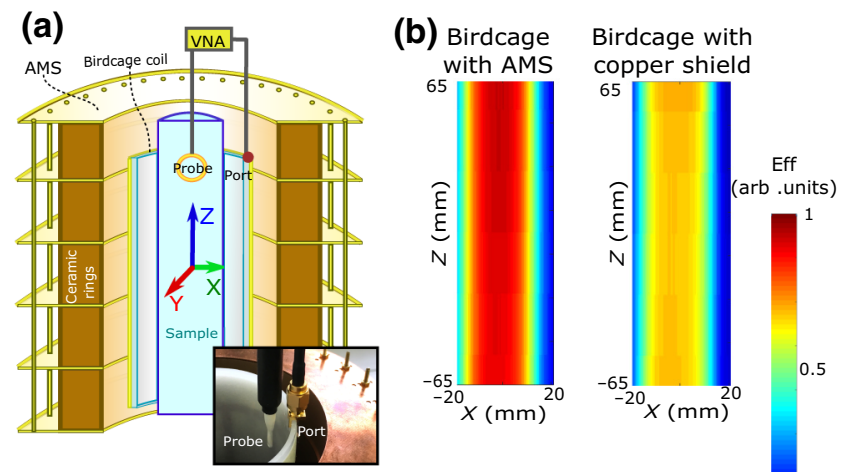

(c)

(d) Birdcage

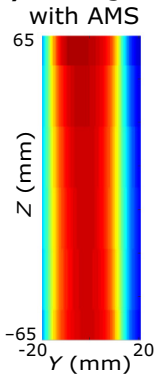

Birdcage with copper shield

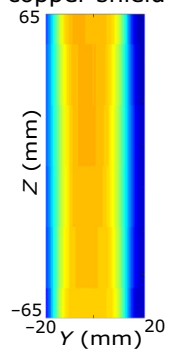

FIG. 8. (a) Schematic illustration of setup for field-probing measurements. (b) Measured efficiency map for PISA and copper shield (reference coil) in $X Z$ plane. (c) Measured efficiency gain. (d) Measured efficiency map for PISA and copper shield (reference coil) in $Y Z$ plane.

of the accepted power, is calculated from the $S$-parameters measured by the VNA as $\left|S_{12}\right| / \sqrt{1-\left|S_{11}\right|^{2}}$. To evaluate the efficiency versus the radius of the sample, we make two cylindrical phantoms from thin-walled polyethylene cans of radii 7.5 and $13.5 \mathrm{~mm}$ filled with a commercially available liquid [35] with the same permittivity and conductivity as in the simulations. In this case, the same formula is used to compare the efficiency, but the $S$ parameters are measured with the probe inserted into the liquid and positioned along the axis of each sample. A schematic illustration of the field-probing experiment and a photograph of the probe near the prototype are shown in Fig. 8(a).

In Figs. 8(b) and 8(d), color maps of the measured efficiency in the $X Z$ and $Y Z$ planes, respectively, are shown for PISA and the reference coil. It is seen that replacing the copper shield with the AMS gives an efficiency improvement of $12.8 \%$ for the empty birdcage, which is the same as in the simulation of the real structure with all material losses included. Moreover, the two coils have the same homogeneous shape of the field pattern. Therefore, an efficiency gain due to the near-field constructive interference effect, without a change in the shape of the field distribution in the birdcage, has been proven without a sample. In the presence of the two prepared samples with radii of 7.5 and $13.5 \mathrm{~mm}$, the gain reduced to $10 \%$ and 

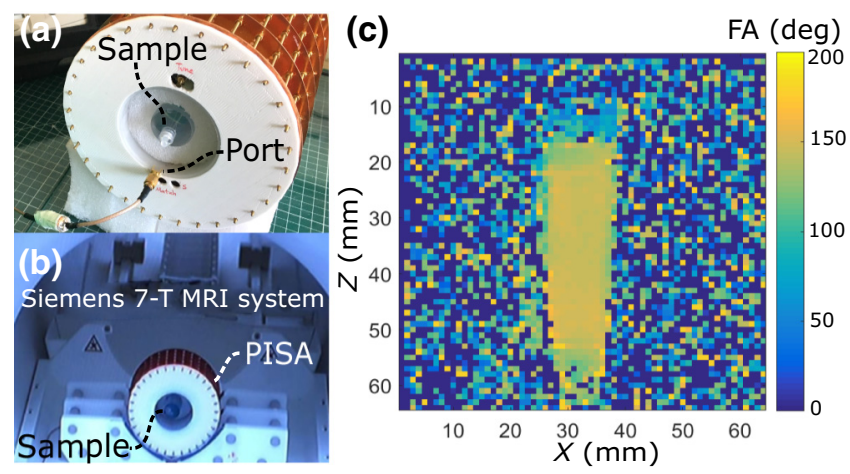

FIG. 9. Test of PISA on 7-T MRI system: (a) assembled prototype with sample inside; (b) PISA placed in the bore of a MRI system; (c) measured FA map, proportional to the main component of the magnetic field in the sample ( $X Z$-plane section) at the ${ }^{19} \mathrm{~F}$ fluorine frequency.

$5 \%$, respectively. From the comparison with the numerical results in Fig. 8(c), one can see that the measured dependence of the gain on the sample radius is similar to our theoretical expectations. For every $R_{\text {sample }}$ considered, there is a good correspondence between the experiment and the simulation for PISA with realistic material losses.

To demonstrate the capability of the AMS to operate under conditions of ultrahigh-field MRI, a prototype of PISA [Fig. 9(a)] is used as a coil in transceiver mode to map the field pattern at $280 \mathrm{MHz}$ (the Larmor frequency of ${ }^{19} \mathrm{~F}$ nuclei), in a Siemens 7-T MRI system (CEA Neurospin, France). The XFL method [36] is used to measure a map of the flip-angle (FA) distribution with a reference voltage of $40 \mathrm{~V}$, which is proportional to the magnetic field produced by the coil during transmission. The coil contains a homogeneous cylindrical sample of radius $27 \mathrm{~mm}$ containing a water-fluorine mixture. In Fig. 9(b), the PISA coil is shown inside the MRI system, while Fig. 9(c) shows the measured FA pattern. Based on Fig. 9(c), one can confirm that the image of the sample is homogeneous. This means that PISA operates correctly in both the transmit and the receive modes in MRI and is capable of imaging ${ }^{19} \mathrm{~F}$.

\section{DISCUSSION AND CONCLUSIONS}

In this paper, we investigate the effect of an artificial magnetic shield on the operation of the most common type of $\mathrm{rf}$ coil for MRI, i.e., the birdcage coil. First, it is demonstrated that the transmit efficiency of a birdcage depends significantly on the size of the scanned conductive sample placed in its center and also on the distance to the copper cylindrical shield surrounding the coil. The best efficiency of the birdcage can be achieved when all transmit power is dissipated in the sample. In the receive mode, this means that the coil receives all noise from the sample. In the case of a small sample or a small gap between the coil and the shield, the transmit power is partially dissipated inside the coil due to the finite conductivity of its conductors. In such conditions, the coil cannot reach its peak efficiency.

Our analytical model and simulations show that an ideal magnetic shield, i.e., a PMC boundary, surrounding the coil significantly increases the magnetic field in the sample per unit current, but also increases the electric field per unit current. Theoretically, in the case of a lossless coil, both the electric and the magnetic field are increased by the same coefficient when a conventional metal shield is replaced with an ideal magnetic one. As a result, a magnetic shield, despite causing near-field constructive interference in the sample, provides no efficiency improvement. Generally, the efficiency in the absence of intrinsic losses (or radiation losses) does not depend on the properties of the shield. Otherwise, in the case of a relatively small sample or a small gap between the coil and the shield, our theory predicts a certain efficiency gain due to an ideal magnetic shield, given by Eq. (19). It is also demonstrated that the gain becomes larger at lower operational frequencies. This can be explained by the fact that the losses in a conductive sample are frequency dependent and always dominate over the intrinsic coil losses at higher frequencies. Therefore, the improvement due to a magnetic shield could be higher in high-field MRI (up to $3 \mathrm{~T}$ ) than in ultrahigh-field MRI ( $7 \mathrm{~T}$ and above). On the other hand, since a magnetic conductor can be approximated only by a resonant structure, operation at very low frequencies is complicated due to the requirement for strong miniaturization of the resonators that need to be contained between the coil and the gradient system of the scanner.

To demonstrate the constructive interference effect for the fields inside a sample, we propose a practical realization of an AMS for a small birdcage 7-T MRI system. We employ an axially symmetric corrugated structure filled with a high-permittivity ceramic material. In contrast to an ideal PMC boundary, the AMS is a finite-sized resonator and operates only due to excitation of a particular resonant mode. Our structure supports multiple coupled modes of five mutually coupled resonant corrugations. The fundamental mode of the AMS corresponds to the in-phase summation of the fields of all of the corrugations, and therefore has a homogeneous field in the center of the resonator, as shown in the inset of Fig. 5(a). This field has a similar distribution to the fundamental mode of the birdcage to be improved. When the birdcage is inserted into the AMS, in the case where both of them resonate at the same frequency, mode hybridization occurs. As a result, the whole coil (referred to as PISA) has two main coupled resonances. The lower-frequency mode implies constructive interference of the near magnetic field of the birdcage and the secondary field created by induced currents in the corrugated structure. By inspecting the calculated field patterns for the AMS and the PMC [Figs. 6(a) and 6(b)], one can ensure that the lossless AMS has an almost identical effect on the field inside the birdcage to that of the 
ideal magnetic shield at the resonance of the even coupled mode. Interestingly, for a relatively small sample, both the ideal and the real magnetic shield provide the same efficiency gain, and the resulting field has the same shape of its distribution. This behavior is also in agreement with the analytical model. Thus, only the efficiency of the coil can be modified, and not the homogeneity of imaging. The only difference from the ideal boundary in terms of the field distribution is observable outside of the birdcage: the magnetic field is resonantly enhanced inside the corrugated structure. It is also observed, both in the simulations and then in the measurements, that there is no change in the axial field distribution. In PISA, the even mode discussed above is tuned to the Larmor frequency of ${ }^{19} \mathrm{~F}$ nuclei at $7 \mathrm{~T}(280 \mathrm{MHz})$ for a further proof-of-principle experiment. The odd coupled mode of PISA leads to destructive interference of the magnetic fields of the coil and the AMS at the center of the sample and therefore is useless in MRI.

Additionally, by numerical simulations, we investigate the effect of losses in the real materials from which the structure is made. Thus, when we consider the dielectric losses in the $\mathrm{CaTiO}_{3}$ rings and the finite conductance of the copper walls of the corrugated structure and its 32 brass rods at the periphery, it is found that the efficiency gain is reduced by almost a factor of $2(13 \%$ instead of $25 \%$ for the geometry considered) but is still noticeable, so that an experimental proof is done. Another important conclusion from the simulations is that radiation losses are negligible for either a copper or an AMS shield. This means that the magnetic shield changes only the ratio between the power absorbed in the sample and the power dissipated in the coil, which in sum give the power accepted from the transmitter [0.5 W in Figs. 6(c) and 6(d) for the simulations].

In the experiment, a PISA exactly similar to the model used in the numerical simulations is compared with the same birdcage with a conventional copper shield (the reference coil). The results precisely confirm the efficiency gain of $13 \%$ for a sample with a radius of $7.5 \mathrm{~mm}$ in the case where the losses of the corrugated structure are included in the simulation. Also, we experimentally demonstrate that the shapes of the magnetic field distributions of the reference coil and PISA are identical in two orthogonal planes. Therefore, in our experiment, the AMS replaces the copper shield when the birdcage is underloaded, resulting in improved efficiency and no modification of the imaging homogeneity. The proper operation of the proposed shield is additionally confirmed by obtaining a phantom image on a 7-T MRI system.

The constructive near-field effect due to the corrugated AMS demonstrated for a birdcage coil has practical importance only in the case of insufficient loading with a conductive sample. In ultrahigh-field MRI methods for biomedical research, poor loading of a birdcage may occur with the relatively small diameter of the head of a volunteer. Also, for preclinical coils, a considerable variation of sample size may take place for laboratory animals such as rodents. Finally, it is possible to translate this concept to clinical MRI at 1.5-3 $\mathrm{T}$ to increase the loading by the patient's body and reduce the losses in the conductors of the coil, which are significant at lower frequencies. For instance, insufficient loading of a whole-body birdcage becomes a problem in clinical MRI when scanning children in a fullsize bore. The proposed method could also be applicable for extending a transmitting birdcage for use with a human head while keeping the efficiency relatively high. Such coils are usually placed very tightly around the object to be imaged, which is uncomfortable, limits the space for placing receive arrays between a head and the birdcage, and leads to a high specific absorption rate (SAR) in local electric field hotspots. However, tight-fitting coils maximize the transmit efficiency if they are properly loaded, that is, the most of the transmit power is dissipated in the sample. With an AMS, it becomes possible to create an extended birdcage coil that is much more comfortable for a patient and creates a lower local SAR but has the same efficiency. Because of the requirement for stronger miniaturization at the frequencies used for clinical MRI, the corrugated structure filled with ceramic presented here cannot be employed at 1.5 and $3 \mathrm{~T}$. Instead, one needs to develop another resonator to be coupled to the birdcage, based on PCB metal structures with structural capacitance or lumped capacitors. Such designs are the subject of future work. Nevertheless, there is an important area for practical application of the effect investigated here to improve the efficiency and, therefore, the imaging quality of MRI.

\section{ACKNOWLEDGMENTS}

The theoretical work and the manufacture were supported by the Russian Science Foundation (Grant No. 18-19-00482). The experimental work received funding from the European Union's Horizon 2020 Research and Innovation Program under Grant No. 736937. The MRI test received financial support from the French "Investissement d'Avenir" program run by the Agence Nationale pour la Recherche, Grant No. ANR-11-INBS-0006 "Infrastructure d'avenir en Biologie Santé," and from the ERPT equipment program of the Leducq Foundation. The authors are thankful to Christopher Collins, Christophe Craeye, Dmitry Dmitriev, and Maxim Konstantinov for useful discussions and to Elizaveta Nenasheva for manufacture of the ceramic rings.

\section{APPENDIX: EXPRESSIONS FOR THE BOUNDARY PROBLEM}

For the boundary problem of a homogenized infinitely long birdcage, we apply five boundary conditions. At the impedance shield ( $\left.\rho=R_{\text {shield }}\right)$, the following surface 
boundary condition is valid for the tangential field components:

$$
E_{z}^{\mathrm{III}}=Z_{S} H_{\varphi}^{\mathrm{III}} .
$$

Next, at the interface between the dielectric material of the sample and the air, i.e., at $\rho=R_{\text {sample }}$, the tangential components of both the $H$ and the $E$ field are continuous:

$$
\begin{gathered}
E_{z}^{\mathrm{I}}=E_{z}^{\mathrm{II}}, \\
H_{\varphi}^{I}=H_{\varphi}^{\mathrm{II}} .
\end{gathered}
$$

Finally, at the surface carrying the equivalent surface current of the homogenized birdcage, the step in the tangential $H$-field component is equal to the given surface current of the source, while the tangential $E$ field is continuous:

$$
\begin{gathered}
H_{\varphi}^{\mathrm{III}}-H_{\varphi}^{\mathrm{II}}=I_{c}, \\
E_{\varphi}^{\mathrm{III}}=E_{\varphi}^{\mathrm{II}} .
\end{gathered}
$$

The five unknown coefficients in the equation for $E_{z}$ [Eq. (6)] can be found using the five boundary conditions
[Eqs. (A1a), (A2a), (A2b), (A3a), and (A3b)], and $H_{\varphi}$ can be calculated using the Maxwell equation $\nabla \times \mathbf{E}=$ $-j \omega \mu_{0} \mathbf{H}$ :

$$
\begin{aligned}
H_{\varphi}^{i}= & -\frac{j \cos \varphi}{\omega \mu_{0}}\left\{k A^{i}\left[J_{0}(k \rho)-\frac{J_{1}(k \rho)}{k \rho}\right]\right. \\
& \left.+k B^{i}\left[Y_{0}(k \rho)-\frac{Y_{1}(k \rho)}{k \rho}\right]\right\} .
\end{aligned}
$$

Substitution of Eqs. (6) and (A4) into the five boundary conditions gives a matrix equation [Eq. (7)], the solution of which can be written in the following form:

$$
\left[\begin{array}{c}
A^{\mathrm{I}} \\
A^{\mathrm{II}} \\
B^{\mathrm{II}} \\
A^{\mathrm{III}} \\
B^{\mathrm{III}}
\end{array}\right]=\underbrace{\left[\begin{array}{lllll}
M_{11} & M_{12} & M_{13} & M_{14} & M_{15} \\
M_{21} & M_{22} & M_{23} & M_{24} & M_{25} \\
M_{31} & M_{32} & M_{33} & M_{34} & M_{35} \\
M_{41} & M_{42} & M_{43} & M_{44} & M_{45} \\
M_{51} & M_{52} & M_{53} & M_{54} & M_{55}
\end{array}\right]^{-1}}_{\overline{\bar{M}}} \cdot\left[\begin{array}{c}
0 \\
0 \\
0 \\
I_{0} \\
0
\end{array}\right] .
$$

The matrix involved is calculated as follows:

$$
\overline{\bar{M}}=\left[\begin{array}{ccccc}
0 & J_{1}\left(k_{1} R_{b}\right) & k_{1} J_{0}\left(k_{1} R_{b}\right)-\frac{J_{1}\left(k_{1} R_{b}\right)}{R_{b}} & 0 & 0 \\
0 & -J_{1}\left(k_{0} R_{b}\right) & -k_{0} J_{0}\left(k_{0} R_{b}\right)+\frac{J_{1}\left(k_{0} R_{b}\right)}{R_{b}} & \frac{j}{\omega \mu_{0}}\left[k_{0} J_{0}\left(k_{0} R_{c}\right)-\frac{J_{1}\left(k_{0} R_{c}\right)}{R_{c}}\right] & J_{1}\left(k_{0} R_{c}\right) \\
0 & -Y_{1}\left(k_{0} R_{b}\right) & -k_{0} Y_{0}\left(k_{0} R_{b}\right)+\frac{Y_{1}\left(k_{0} R_{b}\right)}{R_{b}} & \frac{j}{\omega \mu_{0}}\left[k_{0} Y_{0}\left(k_{0} R_{c}\right)-\frac{Y_{1}\left(k_{0} R_{c}\right)}{R_{c}}\right] & Y_{1}\left(k_{0} R_{c}\right) \\
k_{0} J_{0}\left(k_{0} R_{s}\right)-W J_{1}\left(k_{0} R_{s}\right) & 0 & 0 & -\frac{j}{\omega \mu_{0}}\left[k_{0} J_{0}\left(k_{0} R_{c}\right)-\frac{J_{1}\left(k_{0} R_{c}\right)}{R_{c}}\right] & -J_{1}\left(k_{0} R_{c}\right) \\
k_{0} Y_{0}\left(k_{0} R_{s}\right)-W Y_{1}\left(k_{0} R_{s}\right) & 0 & 0 & -\frac{j}{\omega \mu_{0}}\left[k_{0} Y_{0}\left(k_{0} R_{c}\right)-\frac{Y_{1}\left(k_{0} R_{c}\right)}{R_{c}}\right] & -Y_{1}\left(k_{0} R_{c}\right)
\end{array}\right],
$$

where the parameter $W=1 / R_{s}-j \omega \mu_{0} / Z_{s}$ is introduced. Having calculated the unknown coefficients of the matrix equation [Eq. (7)] by substituting into Eq. (6), it is easy to calculate $E_{z}$ at every point of the entire volume of the coil.

$H_{\varphi}$ can be calculated from Eq. (A4), while $H_{\rho}$ can be defined using the following equation:

$$
H_{\rho}^{i}=-\frac{j \sin \varphi}{\omega \mu_{0} \rho}\left[A^{i} J_{1}(k \rho)+B^{i} Y_{1}(k \rho)\right]
$$

[1] P. B. A. Kumar and W. A. Edelstein, Noise figure limits for circular loop MR coils, Magn. Reson. Med. 61, 1201 (2009).

[2] C. E. Hayes, W. A. Edelstein, J. H. Schenck, O. W. Mueller, and M. Eash, An efficient, highly homogeneous radiofrequency coil for whole-body NMR imaging at $1.5 \mathrm{~T}$, J. Magn. Res. 63, 622 (1985).

[3] F. D. Doty, G. Entzminger, Jr., C. D. Hauck, and J. P. Staab, Practical aspects of birdcage coils, J. Magn. Reson. 138, 144 (1999). 
[4] E. Hayes, The development of the birdcage resonator: A historical perspective, NMR Biomed. 22, 908 (2009).

[5] M. D. Harpen, Radiative losses of a birdcage resonator, Magn. Reson. Med. 29, 713 (1993).

[6] H. Wheeler, Formulas for the skin effect, Proc. IRE 30, 412 (1942).

[7] C. M. Collins, S. Li, Q. X. Yang, and M. B. Smith, A method for accurate calculation of $B_{1}$ fields in three dimensions. Effects of shield geometry on field strength and homogeneity in the birdcage coil, J. Magn. Res. 125, 233 (1997).

[8] J. T. Vaughan and J. R. Griffiths, RF Coils for MRI (John Wiley and Sons, Chichester, United Kingdom, 2012).

[9] P. Kildal, Artificially soft and hard surfaces in electromagnetics, IEEE Trans. Ant. Propag. 8, 1537 (1990).

[10] C. Simovski and A. Sochava, High-impedance surfaces based on self-resonant grids. analytical modelling and numerical simulations, IEEE Antennas and Propag. Soc., APS Int. Symp. (Digest) 4, 434 (2003).

[11] S. A. Tretyakov and C. R. Simovski, Wire antennas near artificial impedance s. urfaces, Microw. Opt. Technol. Lett. 27, 46 (2000).

[12] R. Hansen, Effects of a high-impedance screen on a dipole antenna, IEEE Antennas Wirel. Propag. Lett. 1, 46 (2002).

[13] D. Pozar, Wideband reflectarrays using artificial impedance surfaces, Electron. Lett. 43, 148 (2007).

[14] D. Sievenpiper, Forward and backward leaky wave radiation with large effective aperture from an electronically tunable textured surface, IEEE Trans. Antennas Propag. 53, 236 (2005).

[15] N. Engheta, Thin absorbing screens using metamaterial surfaces, IEEE Antennas Propag. Soc. Int. Symp. 2, 392 (2002).

[16] D. Zhirihin, C. Simovski, P. Belov, and S. Glybovski, Mushroom high-impedance metasurfaces for perfect absorption at two angles of incidence, IEEE Antennas Wirel. Propag. Lett. 16, 2626 (2017).

[17] D. Sievenpiper, L. Zhang, R. J. Broas, N. Alexöpolous, and E. Yablonovitch, High-impedance electromagnetic surfaces with a forbidden frequency band, IEEE Trans. Microw. Theory Tech. 47, 2059 (1999).

[18] A. Feresidis, G. Goussetis, S. Wang, and J. Vardaxoglou, Artificial magnetic conductor surfaces and their application to low-profile high-gain planar antennas, IEEE Trans. Antennas Propag. 53, 209 (2005).

[19] H. Li, J. Hao, L. Zhou, Z. Wei, L. Gong, H. Chen, and C. T. Chan, All-dimensional subwavelength cavities made with metamaterials, Appl. Phys. Lett. 89, 104101 (2006).

[20] Fei-Ran Yang, Kuang-Ping Ma, Yongxi Qian, and T. Itoh, A novel TEM waveguide using uniplanar compact photonic-bandgap (UC-PBG) structure, IEEE Trans. Microw. Theory Tech. 47, 2092 (1999).
[21] Z. Chen, K. Solbach, D. Erni, and A. Rennings, Electromagnetic field analysis of a dipole coil element with surface impedance characterized shielding plate for 7-T MRI, IEEE Trans. Microw. Theory Tech. 64, 392 (2016).

[22] Z. Chen, K. Solbach, D. Erni, and A. Rennings, Improving $B_{1}$ efficiency and signal-to-noise-ratio of a surface coil by a high-impedance-surface RF shield for 7-T magnetic resonance imaging, IEEE Trans. Microw. Theory Tech. 65, 988 (2017).

[23] F. Capolino, Theory and Phenomena of Metamaterials (Taylor \& Francis Group, Boca Raton, USA, 2009).

[24] A. Harvey, Periodic and guiding structures at microwave frequencies, IRE Trans. Microwave Theory Tech. 8, 30 (1959).

[25] C. van Leeuwen, M. Lunenburg, S. Glybovski, P. Luijten, D. Klomp, C. van den Berg, and A. Raaijmakers, in Proc. Int. Soc. Mag. Reson. Med. (Paris, France, 2018).

[26] S. B. Glybovski, V. P. Akimov, and A. E. Popugaev, Analytical study of annular-ring microstrip antennas shorted with thin wires, IEEE Trans. Antennas Propag. 62, 3348 (2014).

[27] H. Vesselle and R. Collin, The signal-to-noise ratio of nuclear magnetic resonance surface coils and application to a lossy dielectric cylinder model. I. theory, IEEE Trans. Biomed. Eng. 42, 497 (1995).

[28] M. I. Kontorovich, V. Y. Petrun'kin, N. A. Esepkina, and M. I. Astrakhan, The coefficient of reflection of a plane electromagnetic wave from a plane wire mesh, Radiotekh. Elektron. 7, 239 (1962).

[29] S. Tretyakov, Analytical Modeling in Applied Electromagnetics (Artech House, Boston, London, 2003).

[30] M. I. Astrakhan, N. M. Zolotukhina, and G. A. Sebyakina, Average boundary conditions for metal grids with conductors of noncircular crosssection, Radiotekh. Elektron. 20, 2417 (1975).

[31] https://www.bruker.com/products/mr/preclinical-mri/mrirf-coils-technical-details.html

[32] E. Nenasheva, L. P. Mudroliubova, and N. F. Kartenko, Microwave dielectric properties of ceramics based on $\mathrm{CaTiO}_{3}-\mathrm{LnMO}_{3}$ system ( $\left.\mathrm{Ln}-\mathrm{La}, \mathrm{Nd} ; \mathrm{M}-\mathrm{Al}, \mathrm{Ga}\right)$, J. Eur. Ceram. Soc. 23, 2443 (2003).

[33] C. Ahn and Z. Cho, Analysis of eddy currents in nuclear magnetic resonance imaging, Magn. Reson. Med. 17, 149 (1991).

[34] F. D. Doty, G. Entzminger, J. Kulkarni, K. Pamarthy, and J. P. Staab, Radio frequency coil technology for smallanimal MRI, NMR Biomed. 20, 304 (2007).

[35] https://www.mvg-world.com/sites/default/files/2019-09/

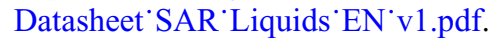

[36] A. Amadon, M. A. Cloos, N. Boulant, M. Hang, C. J. Wiggins, and H. Fautz, in Proceedings of the International Society for Magnetic Resonance in Medicine (Montréal, Canada, 2012), p. 4885. 